\title{
Produção Científica sobre Educação Médica no Brasil: Estudo a partir das Publicações da Revista Brasileira de Educação Médica
}

\author{
Scientific Production on Medical Education in \\ Brazil: a Study Based on the Brazilian \\ Journal of Medical Education
}

Pedro Tadao Hamamoto Filho

Carlos Alberto Santos Filho Joélcio Francisco Abbade José Carlos Peraçoli

\section{PALAVRAS-CHAVE \\ - Educação Médica; \\ - Pesquisa em Educação Médica; \\ - Produção Científica.}

- Medical Education;

- Research in Medical Education;

- Scientific Production.

Recebido em: 13/10/2012

Reencaminhado em: 29/11/2012

Reencaminhado em: 20/03/2013

Reencaminhado em: 20/05/2013

Aprovado em: 28/08/2013

\section{ABSTRACT}

Research in medical education is the critical and systematic study of teaching and learning in medicine. It's a developing field with an increasing number of researchers and publications. In Brazil, the Brazilian Journal of Medical Education (RBEM) is the main journal in the area. The present study describes Brazilian scientific production in medical education by analyzing papers published by the RBEM from 2006 to 2010. In this period, the number of articles increased by 117.6\%, averaging 60.4 articles per year. There was also an increase in the proportion of new research studies. The Southeast region and public institutions account for the greatest share of the articles. The most frequent keywords were "medical education", "medical students" and "curriculum". The growth of the RBEM reflects the development of research in medical education in Brazil and is in keeping with the logic of national scientific production. 


\section{INTRODUÇÃO}

Os professores de Medicina tendem a procurar as melhores evidências científicas para tomada de decisões clínicas, principalmente com o advento e o respaldo da Medicina Baseada em Evidências. Por outro lado, nem sempre adotam essa postura em relação à sua atividade docente, como na avaliação de alunos e abordagens pedagógicas ${ }^{1,2}$. Se os pesquisadores em educação médica desejam transformar o ensino médico, é necessário que a pesquisa seja acessível, compreensível, convincente e claramente relacionada à prática dos médicos-professores ${ }^{3}$.

A pesquisa em educação médica teve início na década de 1950 com George Miller, na Universidade do Estado de Nova York, Buffalo (NY). Foram seus os primeiros esforços para, através da pesquisa, qualificar o ensino e aprendizagem nas escolas médicas de modo sustentado e com financiamento. Desde então, a pesquisa em educação médica é motivação dos profissionais da saúde interessados em enriquecer o ambiente de ensino ${ }^{4}$.

Assim, a pesquisa em educação médica tornou-se um estudo crítico e sistemático do ensino e aprendizagem em Medicina, incluindo análises de processos e resultados em todas as fases da formação profissional ${ }^{5}$. Trata-se de um campo em crescimento qualitativo e quantitativo, como se pode observar pelo número de pessoas envolvidas e de produções geradas 4 .

Pela importância do tema, em 1999, a conferência anual da Association for Medical Education in Europe (Amee), realizada na Suécia, criou uma iniciativa de fortalecimento da pesquisa em educação médica, chamada Best Evidence in Medical Education (Beme), cujo objetivo é apontar as melhores evidências em educação médica com base em estudos publicados na área ${ }^{6}$

De fato, o número de publicações na área cresceu consideravelmente nos últimos 20 anos. Até a década de 1980, existiam três grandes periódicos afins: Academic Medicine, Medical Education e Medical Teacher, editados, respectivamente, pela Association of American Medical Colleges, Association for the Study of Medical Education e Amee. Em 1989, foi lançado o Teaching and Learning in Medicine. Em 1990, o Academic Medicine reservou um suplemento especial para a publicação de apresentações da conferência de pesquisa em educação médica da AAMC, e em 1996 teve início a publicação do Advances in Health Sciences Education. Finalmente, em 1999, o Medical Education aumentou sua periodicidade de 6 para 12 fascículos por $\mathrm{ano}^{7}$.

No Brasil, ainda que timidamente, também ocorreram avanços. Em 1997, é lançada a primeira edição da revista Interface - Comunicação, Saúde e Educação, pela Fundação UNI/
Botucatu, que atualmente está indexada na Scopus. Em 2006, a Revista Brasileira de Educação Médica (RBEM), editada pela Associação Brasileira de Educação Médica (ABEM), foi indexada ao SciELO, estimulando pesquisadores à publicação de qualidade na área ${ }^{8}$.

Estas considerações nos levam a perguntar: como está a pesquisa em educação médica no Brasil? De fato, ela vem se desenvolvendo? Onde e por quem é produzida? Diante dessas indagações, a proposta deste estudo foi caracterizar a pesquisa em educação médica brasileira com base nas publicações da RBEM com relação à quantidade de artigos publicados, às seções de publicação, à procedência geográfica e institucional dos autores e aos descritores mais utilizados.

\section{METODOLOGIA}

Foi realizada uma análise descritiva dos artigos publicados pela RBEM, disponibilizados gratuitamente no SciELO (www. scielo.org) no período de 2006 a 2010, excluindo-se editoriais e erratas. Os artigos foram avaliados de acordo com o ano de publicação, a seção da revista, a procedência institucional e geográfica dos autores e os descritores utilizados.

Em relação à procedência institucional, foram considerados os autores com vínculo exclusivo em instituição pública, exclusivo em instituição privada ou autores com duplo vínculo.

Na distribuição dos autores por procedência geográfica, foram consideradas as unidades da Federação e regiões do País.

\section{RESULTADOS}

No período estudado, a RBEM publicou 302 artigos assim distribuídos: 34 em 2006, 39 em 2007, 64 em 2008, 91 em 2009 e 74 em 2010. Verifica-se um crescimento numérico de 117,6\% entre 2006 e 2010, com uma média de 60,4 artigos por ano, podendo-se afirmar que esse crescimento está relacionado ao aumento de três para quatro fascículos anuais a partir de 2008. Identificaram-se oito seções de publicação: pesquisas inéditas, ensaios, relatos de experiência, documentos, resenhas, resumos de tese, revisões e temas globais. As três primeiras foram as mais frequentes e juntas correspondem a mais de $90 \%$ dos artigos.

As pesquisas inéditas eram responsáveis por cerca de $50 \%$ a $60 \%$ das publicações entre 2006 e 2008 . A partir de 2009 , observa-se crescimento desta proporção, chegando a $71,6 \%$ em 2010. Em média, foram publicadas 37,6 pesquisas originais por ano. Os relatos de experiência tiveram participação percentual que variou de $5,5 \%$ a $25 \%$. Os ensaios representaram $12,9 \%$ das publicações (Tabela 1). 
TABELA 1

Número de artigos publicados pela RBEM por seção e ano (de 2006 a 2010)

\begin{tabular}{|c|c|c|c|c|c|c|c|c|c|c|c|c|}
\hline \multirow{2}{*}{ Seção } & \multicolumn{2}{|c|}{2006} & \multicolumn{2}{|c|}{2007} & \multicolumn{2}{|c|}{2008} & \multicolumn{2}{|c|}{2009} & \multicolumn{2}{|c|}{2010} & \multicolumn{2}{|c|}{ Total } \\
\hline & $\mathrm{n}$ & $\%$ & $\mathrm{n}$ & $\%$ & $\mathrm{n}$ & $\%$ & $\mathrm{n}$ & $\%$ & $\mathrm{n}$ & $\%$ & $\mathrm{n}$ & $\%$ \\
\hline Pesquisa & 20 & 58,8 & 22 & 56,4 & 32 & 50,0 & 61 & 67,0 & 53 & 71,6 & 188 & 62,3 \\
\hline Ensaio & 5 & 14,7 & 4 & 10,3 & 8 & 12,5 & 16 & 17,6 & 6 & 8,1 & 39 & 12,9 \\
\hline $\begin{array}{l}\text { Relato de } \\
\text { experiência }\end{array}$ & 6 & 17,7 & 5 & 12,8 & 16 & 25,0 & 5 & 5,5 & 14 & 18,9 & 46 & 15,2 \\
\hline Resenha & 0 & 0,0 & 2 & 5,1 & 3 & 4,7 & 4 & 4,4 & 1 & 1,6 & 10 & 3,3 \\
\hline Res. tese & 2 & 5,9 & 3 & 7,7 & 2 & 3,1 & 2 & 2,2 & 0 & 0,0 & 9 & 3,0 \\
\hline Revisão & 0 & 00 & 1 & 2,6 & 3 & 4,7 & 3 & 3,3 & 0 & 0,0 & 7 & 2,3 \\
\hline Tema global & 0 & 0,0 & 2 & 5,1 & 0 & 0,0 & 0 & 0,0 & 0 & 0,0 & 2 & 0,7 \\
\hline Documento & 1 & 2,9 & 0 & 0,0 & 0 & 0,0 & 0 & 0,0 & 0 & 0,0 & 1 & 0,3 \\
\hline
\end{tabular}

Identificamos 1.067 autores (média de 3,5 por artigo). Observamos a presença de pelo menos um autor do Estado de São Paulo em 126 dos 302 artigos. Em 59 artigos há autores do Estado do Rio de Janeiro e, em ordem decrescente de frequência, aparecem autores do Paraná, Minas Gerais, Rio Grande do Sul, Santa Catarina e Distrito Federal (Gráfico 1).

\section{GRÁFICO 1}

Número de artigos com que cada unidade da Federação está representada nas edições da RBEM (excluídos editoriais e erratas; de 2006 a 2010)

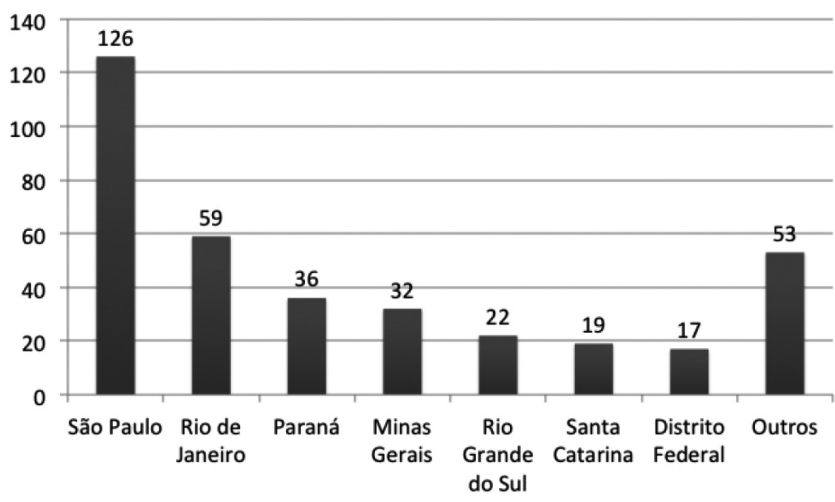

Assim, a Região Sudeste teve a maior participação, contribuindo em 218 artigos, seguida das regiões Sul (77 artigos), Centro-Oeste e Nordeste (32 artigos cada) e Norte (5 artigos).

Autores estrangeiros participaram de nove artigos, sendo quatro participações de Portugal, duas do Chile e uma da Argentina, de Angola e da Holanda.
Com relação ao vínculo institucional, em 254 artigos $(84,1 \%)$ os autores se declararam vinculados exclusivamente a instituições públicas, e em $26(8,6 \%)$, a instituições privadas. Em 22 artigos (7,3\%) havia colaboração de autores de instituições públicas e privadas ou autores de duplo vínculo. A Tabela 2 detalha a vinculação institucional dos autores de acordo com a seção de publicação de seus artigos.

TABELA 2

Percentual de autores participantes dos artigos da RBEM segundo tipo de vínculo e seções de maior publicação (de 2006 a 2010)

\begin{tabular}{lccc}
\hline Seção & $\begin{array}{c}\text { Exclusivamente } \\
\text { público (\%) }\end{array}$ & $\begin{array}{c}\text { Exclusivamente } \\
\text { privado (\%) }\end{array}$ & $\begin{array}{c}\text { Público/ } \\
\text { privado (\%) }\end{array}$ \\
\hline Pesquisa & 73,4 & 8,5 & 18,1 \\
Ensaio & 66,7 & 7,7 & 25,6 \\
$\begin{array}{l}\text { Relato de } \\
\text { experiência }\end{array}$ & 73,9 & 15,2 & 10,9 \\
\hline
\end{tabular}

Autores de vínculo exclusivamente público tiveram mais artigos publicados em todas as seções de publicação. A maior participação relativa de autores de vínculo privado se deu nos relatos de experiência.

Foram identificados 1.143 descritores (média de 3,8 por artigo). Os descritores mais frequentemente utilizados foram educação médica, estudantes de Medicina, currículo e ensino (Gráfico 2). 
GrÁfico 2

Frequência de descritores mais comuns nos artigos da RBEM (de 2006 a 2010)

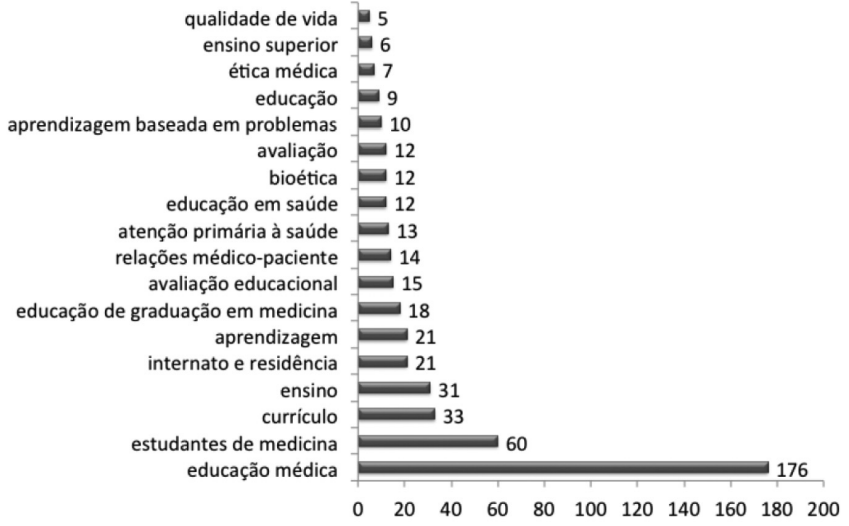

\section{DISCUSSÃO}

Na literatura mundial, a evolução da pesquisa em educação médica está distribuída em três gerações. Após seu surgimento, com George Miller na década de 1950, a primeira geração (década de 1960) era constituída por professores de Medicina que deixaram suas áreas de especialidade médica de origem para se dedicarem à educação. À época, não tinham habilidades e conhecimentos necessários à educação, como formação em estatística, psicometria e metodologia qualitativa. A estes, na década de 1970, juntaram-se ingleses e holandeses com pós-graduação formal em Psicologia, iniciando suas contribuições à área.

A segunda geração (década de 1990) recrutou profissionais de outras áreas do conhecimento, como psicólogos, antropólogos e sociólogos, para desenvolverem trabalhos no meio médico. Esse movimento enriqueceu a pesquisa em educação médica com variações sofisticadas de metodologias de pesquisa e fundamentação teórica.

Já a terceira geração, contemporânea, tem formado profissionais para dedicação à educação médica, com maior independência de outras especialidades na concepção e condução de projetos ${ }^{2}$.

No Brasil, presenciamos a consolidação desta formação. Como exemplo, podem-se citar iniciativas da Coordenação de Aperfeiçoamento de Pessoal de Nível Superior (Capes) por meio da implantação de programas de pós-graduação em educação em saúde e do fomento para linhas de pesquisa de ensino em programas de pós-graduação já estruturados ${ }^{9,10}$. Cite-se também o programa de desenvolvimento docente financiado pelo Ministério da Saúde e executado pelo Instituto Faimer Brasil (Foundation for Advancement of International Medical Education and Research).
Como resultado do crescimento do número de pesquisadores em atividade e em formação para a educação médica, vivencia-se o aumento de publicações na área. $\mathrm{O}$ crescimento quantitativo de artigos publicados pela RBEM espelha o desenvolvimento da produção científica em educação médica no Brasil. Prova disso é o aumento de três para quatro fascículos anuais da revista em 2008. O pico de publicações em 2009 aconteceu devido à publicação de um suplemento com artigos do projeto de avaliação de tendências de mudanças no curso de graduação nas escolas pela Comissão de Avaliação das Escolas Médicas (Caem) da ABEM.

Há que se considerar esse crescimento da produção científica em educação médica no contexto do crescimento da produção científica brasileira em geral. O Brasil aumenta não apenas seu número de publicações nacionais e internacionais, como também sua contribuição proporcional na produção científica mundial ${ }^{11,12}$. Este aumento, aliás, deve muito à participação das ciências biológicas e da saúde ${ }^{13,14}$.

Parece-nos haver também um amadurecimento da produção científica. Se entre 2006 e 2008 as pesquisas inéditas representavam de $50 \%$ a $60 \%$ das publicações da RBEM, nos anos de 2009 e 2010 elas representaram 67\% e 71,6\% respectivamente. Ainda que se fundamente em um período curto, esta análise aponta maior publicação de experimentos em educação médica nos últimos dois anos do período estudado. Posto de outra forma, publicou-se proporcionalmente menor número de relatos de experiências institucionais singulares e maior número de pesquisas com método científico.

Com relação à procedência dos autores, repete-se a lógica da produção científica nacional, isto é, predomínio da Região Sudeste - São Paulo em particular - e de instituições públicas. Isto está certamente relacionado à tradição dos programas de pós-graduação e pesquisa das instituições estaduais e federais da Região Sudeste, que fomentam cenários de produção científica em geral e, por conseguinte, também no âmbito da educação médica.

Neste sentido, vale ressaltar a maior participação proporcional das instituições privadas nos relatos de experiência e menor nas pesquisas inéditas, corroborando os achados de que a produção científica de ponta, de maior maturidade intelectual, emana de instituições públicas. Porém deve-se ressalvar que a revista não lista todas as possíveis vinculações dos autores, mas somente aquelas declaradas quando da submissão do artigo. Mas em geral declaram-se aquelas em que os autores desenvolveram o trabalho que deu origem à publicação. Portanto, a maior parte dos trabalhos parece mesmo provir de instituições públicas.

A frequência dos descritores mais utilizados nos artigos suscita reflexões cuidadosas. "Educação médica" foi o termo 
mais utilizado. Parece-nos redundante que artigos publicados em periódico com o título de educação médica utilizem "educação médica" como descritor. O fato de o artigo ser publicado nesta revista já não é suficiente para caracterizá-lo como de educação médica? Por que os autores insistem em utilizá-lo? Formulamos a hipótese de que os autores ainda não consideram a educação médica como área legitimada ou consagrada do conhecimento e, por isso, utilizam o termo para dar visibilidade aos seus trabalhos em uma área em começo de desenvolvimento.

De acordo com Bligh e Brice $^{15}$, existe a visão de que a pesquisa em educação médica é mais uma ciência médica do que social, o que se demonstra pelas bases de dados em que são indexados os periódicos de educação médica. Nessas bases de dados, portanto, enfatizar a publicação como de "educação médica" pode ser uma necessidade dos autores.

Outros descritores mais utilizados — "estudantes de Medicina", "currículo" e "ensino" — sugerem que as metodologias empregadas nas pesquisas investigam mudanças curriculares e métodos de ensino com base na opinião dos estudantes ou no desempenho de estudantes em avaliações. Esta sugestão deve ser comprovada posteriormente com análises de conteúdo das publicações. Ainda assim, essa sugestão é coerente com a literatura internacional ${ }^{16}$.

Prytowsky e Bordage analisaram quase 600 artigos do Academic Medicine, Medical Education e Teaching and Learning in Medicine e mostraram que, em termos de participantes, aprendizes foram os mais estudados (68,9\% dos artigos), seguidos de professores (19,4\%). Em termos de resultados de análise, desempenho e satisfação, foram os mais frequentes $(49,4 \%$ e $34,1 \%$ dos artigos, respectivamente). Assim, avaliação do desempenho e satisfação de aprendizes estiveram presentes em cerca de $60 \%$ dos artigos analisados ${ }^{17}$.

Achados semelhantes foram descritos por Todres et al. ${ }^{18}$, que, em seu levantamento de artigos publicados no British $\mathrm{Me}$ dical Journal, Medical Education e Medical Teacher, encontraram que $54 \%$ usaram questionários e mais de $70 \%$ tinham como temas mais abordados: avaliação do estudante, desenho curricular, desempenho profissional, características dos estudantes e métodos de ensino.

A literatura, porém, advoga a necessidade de reconhecer as limitações de resultados focados nos estudantes e de avançar para pesquisas que vinculem processos educacionais com resultados para pacientes e seu cuidado ${ }^{19,20}$.

\section{LIMITAÇÕES DO ESTUDO}

Naturalmente, ater-se apenas às publicações da RBEM para caracterizar a pesquisa em educação médica brasileira descon- sidera que outros periódicos também tenham importância na publicação da área. No entanto, levantamento prévio apontou a RBEM como o mais importante e específico periódico para publicação da pesquisa em educação médica brasileira, sem desconsiderar outras revistas que também publicam na área, como a Revista da Associação Médica Brasileira, o São Paulo Medical Journal, a Ciência E Saúde Coletiva, e a Interface (que não se limita à educação médica, mas abrange educação e práticas em outras áreas da saúde) $)^{21}$.

Não foi feita a análise da procedência exata dos 1.067 autores, pois esta informação poderia não ser tão útil. Por exemplo: se houvesse um artigo assinado por cinco autores do Paraná e três artigos assinados por um autor de Minas Gerais cada um, contabilizaríamos cinco autores do Paraná e três de Minas Gerais. Pareceu-nos mais importante a informação de que o Paraná participou de um artigo, e Minas Gerais, de três. Assim, os dados não permitem identificar precisamente o número de autores por unidade da Federação.

Com relação ao vínculo dos autores, não se pode dizer que a maioria dos autores tenha vínculo exclusivamente público. Trata-se de um viés de nosso estudo, dado que a revista publica apenas o vínculo declarado dos autores quando da submissão dos textos. No entanto, o entendimento de que os autores declaram a instituição em que desenvolveram seu trabalho parece-nos permitir o entendimento de que nas instituições públicas se realiza a maioria dos trabalhos em educação médica.

Nosso estudo também não realizou análise de conteúdo dos artigos nem de metodologias empregadas nas pesquisas inéditas, o que forneceria dados para uma análise qualitativa. No entanto, cremos que os dados do presente estudo trazem contribuições que podem suscitar reflexões sobre o tema e apontar perspectivas de novas investigações.

\section{CONCLUSÕES}

O crescimento da RBEM espelha o desenvolvimento da pesquisa em educação médica brasileira, principalmente por instituições públicas do Sudeste do País, num contexto de crescimento da produção científica nacional segundo o mesmo paradigma de instituições. Pela frequência dos descritores utilizados, acreditamos que as metodologias empregadas investigam intervenções educacionais com base na opinião/ satisfação/desempenho dos estudantes.

Para a qualificação da pesquisa em educação médica brasileira, há que se considerar investigações na fronteira do conhecimento, inovadoras, criativas, com forte embasamento teórico, com metodologias adequadas às propostas de pesquisa e focadas na atenção à saúde dos pacientes. 


\section{REFERÊNCIAS}

1. Bligh J, Anderson MB. Medical teachers and evidence. Med Educ. 2000;34:162-3

2. Bligh J. Research in medical education at the start of the century. Med Educ. 2002;36:1000-1. DOI: 10.1046/j.1365-2923.2002.01324_1.x

3. Petersen S. Time for evidence based medical education. BMJ. 1999;318:1223-4. DOI: 10.1136/bmj.318.7193.1223

4. Norman G. Fifty years of medical education research: waves of migration. Med Educ. 2011;45(8):785-91. DOI: 10.1111/j.1365-2923.2010.03921.x

5. Bligh J, Parsell G. Research in medical education: finding its place. Med Educ. 1999;33(3):162-3.

6. Hart IR, Harden RM. Best Evidence Medical Education (BEME): a plan for action. Med Teach. 2000;22(2):131-4.

7. Albert M, Hodges B, Regehr G. Research in medical education: balancing service and Science. Adv in Health Sci Educ. 2007;12:103-15.

8. Martins MA. Editorial. Rev Bras Educ Med. 2006;30(2):3.

9. Manfroi WC, Machado CLB, Dorneles MA, Ribeiro EC, Bordin R. Estratégias para a implementação de um projeto de pós-graduação em Educação e Saúde na Faculdade de Medicina de Universidade Federal do Rio Grande do Sul. Rev Bras Educ Med. 2008;32(1):127-32.

10. Brasil. Ministério da Educação. Coordenação de Aperfeiçoamento de Pessoal de Nível Superior - CAPES. Edital no 24/2010. Pró-Ensino na Saúde. Diário Oficial da União. Brasília, 7 mai. 2010. Seção 3, p. 52. Disponível em: <http:/ / capes.gov.br/images/stories/download/bolsas / Edital_EnsinoSaude_2010.pdf> Acesso 9 fev. 2012.

11. Fundação de Amparo à Pesquisa do Estado de São Paulo. Análise da produção científica a partir de publicações em periódicos especializados. In: Indicadores de Ciência, Tecnologia e Inovação em São Paulo - 2010. São Paulo, 2010. V. 1 , cap 4 .

12. Zorzetto R, Razzouk D, Dubugras MTB, Gerolin J, Schor N, Guimarães JA et al. The scientific production in health and biological sciences of the top 20 Brazillian universities. Braz J Med Biol Res. 2006;39(12):1513-20.

13. Guimarães JA. A pesquisa médica e biomédica no Brasil. Comparações com o desempenho científico brasileiro e mundial. Ciênc Saúde Coletiva. 2004;9(2):303-27.
14. Muccioli C, Campos M, Goldchmit M, Dantas PEC, Bechara SJ, Costa VP. A Produção Científica no Brasil. Arq Bras Oftalmol. 2007;70(4):571.

15. Bligh J, Brice J. What is the value of good medical education research? Med Educ. 2008; 42:652-3.

16. Dimitroff A, Davis WK. Content analysis of research in undergraduate medical education. Acad Med. 1996;71:60-7.

17. Prytowsky JB, Bordage G. An outcomes research perspective on medical education: the predominance of trainee assessment and satisfaction. Med Educ. 2001; 35:331-6.

18. Todres M, Stephenson A, Jones R. Medical education research remains the poor relation. BMJ. 2007;335:333-5.

19. Dauphinee WD, Wood-Dauphinee S. The need for evidence in medical education: the development of best evidence medical education as an opportunity to inform, guide and sustain medical education research. Acad Med. 2004; 79:925-30.

20. Chen FM, Burstin H, Huntington J. The importance of clinical outcomes in medical education research. Med Educ. 2005; 350-1.

21. Hamamoto Filho PT, Santos Filho CA, Peraçoli JC, Abbade JF. Levantamento das publicações brasileiras em educação médica. Rev Bras Educ Med. 2010; 34(3 supl 1):312.

\section{CONTRIBUIÇÃO DOS AUTORES}

Pedro Tadao Hamamoto Filho contribuiu com a concepção e desenho do estudo, tabulação e análise dos dados e redação do texto. Carlos Alberto Santos Filho contribuiu com a extração dos dados e a revisão do texto. Joélcio Francisco Abbade e José Carlos Peraçoli contribuíram com a orientação dos trabalhos e a revisão do texto.

\section{CONFLITO DE INTERESSES}

Declarou não haver.

\section{ENDEREÇO PARA CORRESPONDÊNCIA}

Pedro Tadao Hamamoto Filho

Faculdade de Medicina de Botucatu (Unesp)

Departamento de Neurologia, Psicologia e Psiquiatria

Distrito de Rubião Jr, s/n ${ }^{\circ}$ - Botucatu

CEP 18618-970 - SP

E-mail: pthamamotof@hotmail.com 